\title{
Experimental In-Vivo Models Used in Fat Grafting Research for Volume Augmentation in Soft Tissue Reconstruction
}

\author{
Jorge Lujan-Hernandez, Raghu Appasani, Kylee Sullivan, Leah Siegel-Reamer, Janice F. Lalikos \\ Division of Plastic Surgery, University of Massachusetts Medical School, Worcester, MA, USA
}

As the popularity of fat grafting research increases, animal models are being used as the source of pre-clinical experimental information for discovery and to enhance techniques. To date, animal models used in this research have not been compared to provide a standardized model. We analyzed publications from 1968-2015 to compare published accounts of animal models in fat grafting research. Data collected included: species used, graft characteristics (donor tissue, recipient area, amount injected, injection technique), time of sacrifice and quantification methods. Mice were most commonly used (56\% of studies), with the "athymic nude" strain utilized most frequently (44\%). Autologous fat was the most common source of grafted tissue (52\%). Subcutaneous dorsum was the most common recipient site (51\%). On average, $0.80 \pm 0.60 \mathrm{~mL}$ of fat was grafted. A single bolus technique was used in $57 \%$ of studies. Fat volume assessment was typically completed at the end of the study, occurring at less than 1 week to one year. Graft volume was quantified by weight (63\%), usually in conjunction with another analysis. The results demonstrate the current heterogeneity of animal models in this research. We propose that the research community reach a consensus to allow better comparison of techniques and results. One example is the model used in our laboratory and others; this model is described in detail. Eventually, larger animal models may better translate to the human condition but, given increased financial costs and animal facility capability, should be explored when data obtained from small animal studies is exhausted or inconclusive.

Keywords Models, animal / Research / Research design / Adipose tissue / Fat grafting

\author{
Correspondence: \\ Jorge Lujan-Hernandez \\ Division of Plastic Surgery, University \\ of Massachusetts Medical School, 55 \\ Lake Ave N., Worcester, MA 01655, \\ USA \\ Tel: +1-508-856-3723 \\ Fax: +1-508-856-5250 \\ E-mail: Jorge.Lujan-Hernandez@ \\ umassmemorial.org
}

We would like to acknowledge Heather Tessier, MA, for her editorial contributions.

No potential conflict of interest relevant to this article was reported.

Received: 10 Mar 2017 • Revised: 17 Apr 2017 • Accepted: 26 Apr 2017

pISSN: 2234-6163 • elSSN: 2234-6171 • https://doi.org/10.5999/aps.2017.44.5.361 • Arch Plast Surg 2017;44:361-369

\section{INTRODUCTION}

The technique of fat grafting was first used by Neuber in 1893 and later evolved as a method to repair soft tissue defects [1]. This minimally invasive procedure increases soft tissue volume with minimal trauma, rapid recovery and immediate results. Over the years, the unpredictable reabsorption, formation of calcifications and potential risk of inducing malignancy lead to some concerns and a decline in its usage [1]. However, in the last two decades, despite these concerns, fat grafting has become a valuable resource in the plastic surgeon's armamentarium not only to provide soft tissue volume but also to regenerate damaged tissue by taking advantage of the mesenchymal stem cell population within the graft $[2,3]$. 
While the majority of investigations are showing fat grafting to be safe from an oncologic perspective over the available periods of study $[4,5]$, the optimal technique for the procedure has not been established. There are currently no gold standards for addressing harvesting, processing, re-injecting and recipient site specifications to achieve long-lasting and predictable results [6]. As the prevalence of fat grafting increases in both cosmetic and reconstructive surgery (either as low-volume or high-volume fat grafting), it is crucial that techniques are optimized. Scientific innovation is the pathway to the best procedural methods which is paramount to the future of fat grafting in plastic surgery practice $[7,8]$.

Multiple research groups around the world are actively investigating questions related to adipose tissue and fat grafting. Before beginning human clinical research, preclinical translational studies must be completed. Ideally, methods used in in-vivo and invitro studies should be consistent and systematic for comparison. Inconsistency in translational models makes the results difficult to evaluate, compare and correlate to the human condition. With regards to fat grafting, standardized universal models have not been established. Not only are scientists investigating questions about fat grafting but they are also investigating the methods that they are using to answer those questions.

This paper compiles the current information available on fat grafting translational models as a systematic review. It aims to describe the differences and similarities between published studies and discuss future perspectives.

\section{METHODS}

A comprehensive PUBMED literature review was performed with the search terms "Fat Grafting” AND OR "Fat Graft” published up to April 2015. Each article was screened based on the abstract content and selected if animal models were involved in the study. Then a secondary screening was performed. Only original research articles written in English language and related to fat grafting for soft tissue reconstruction and volume augmentation were included. Review articles were excluded.

Full articles meeting the inclusion criteria were carefully analyzed by 3 reviewers (J.L., R.A., and K.S.) to find commonalities. Factors such as number of publications in the last few years, animal species used in these studies, type of graft (human xenografts, autograft, allograft, or other xenograft), duration of study until sacrifice (reported in weeks), method of volume quantification, graft recipient site, grafting technique, and amount of injection were collected. Data regarding harvesting and processing methods was not included in this review which was focused only on variables pertinent to the animal models used.
Data was pooled and descriptive statistics were obtained from these results and illustrated in graphs (Fig. 1).

\section{RESULTS}

The search yielded 1,408 results published from years 1928 to 2015 related to fat grafting. We then excluded in-vitro, clinical and review papers yielding to 202 results employing fat grafting for a variety of purposes. Of those, we found that only 100 publications were specifically oriented to volume restoration in animal models and were written in English Language. These articles were published between 1968-2015. We found a consistent increase in the number of publications per year in the last 15 years, and a 9-fold increase when comparing years 2000 to 2014 (Fig. 2).

\section{Animal models}

Results demonstrated that the animal model used most commonly was the mouse ( $56 \%$ of the studies). In descending order of popularity, the other species used were rats, rabbits, pigs and dogs (Fig. 3A). Within mice, 7 strains were used fairly consistently, most of them with some level of immunocompromise. $14 \%$ of the studies reported to use wild type (WT B6/C57) mice for studying allo- or autografts. From the immunosup-

\section{Fig. 1. Pubmed literature search}

A pubmed search was done using "Fat Grafting" AND OR "Fat Graft" key words. Inclusion criteria were studies using animals, articles written in English and articles that studied fat grafting used for volume restoration. In vitro, clinical studies and literature reviews were excluded, as well as papers not written in English and not related to volume retention. 100 Studies met our inclusion criteria and were obtained and carefully analyzed by 3 authors.

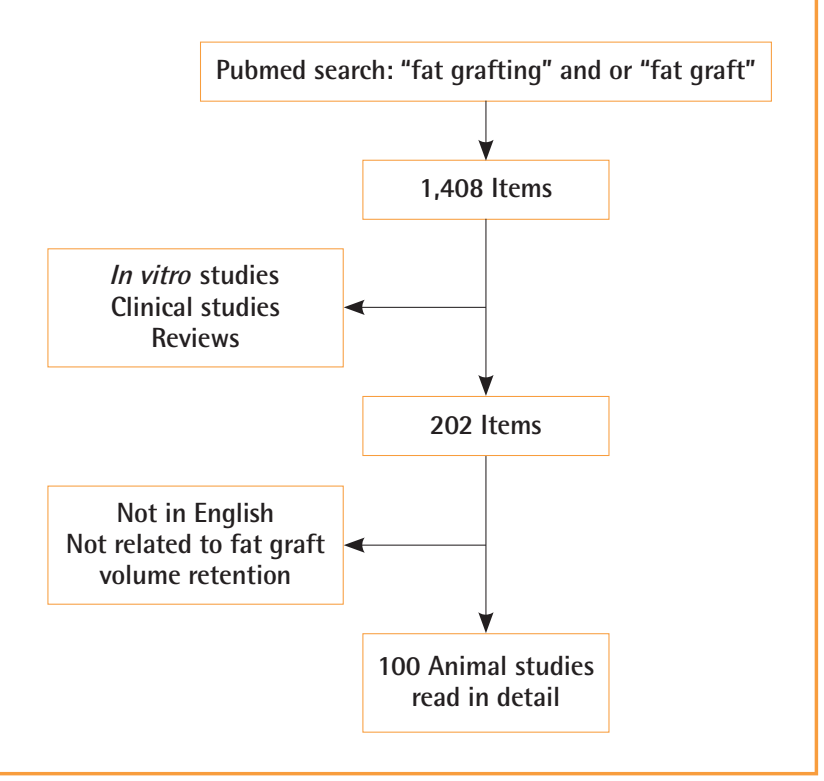


pressed strains, "Athymic Nude" was used more commonly (50\%). These mice lack a thymus, and therefore have no mature $\mathrm{T}$ cells while still preserving the rest of the immune system. $4 \%$ of the studies used the NOD-SCID strain which have impaired $\mathrm{B}$ and $\mathrm{T}$ lymphocytes and deficient natural killer (NK) cells (Fig. 3B).

\section{Type of graft}

$52 \%$ of the studies used autologous grafts (i.e., own inguinal fat pad to dorsum), versus $41 \%$ of studies performing xenografts using human fat. The rest did either allografts or other non-human xenografts (Fig. 4A). Subcutaneous dorsum was the most common recipient site (51\%) with $28 \%$ grafting underneath the scalp. Intra-abdominal (12\%) and head \& neck (9\%, cheeks, ears, neck) grafts were preferred by some studies (Fig. 4B).

\section{Fig. 2. Publications related to fat grafting in the last years}

Of the articles selected, we found an increasing trend of articles published related to fat grafting over the last few years, showing the growing interest of the medical community.

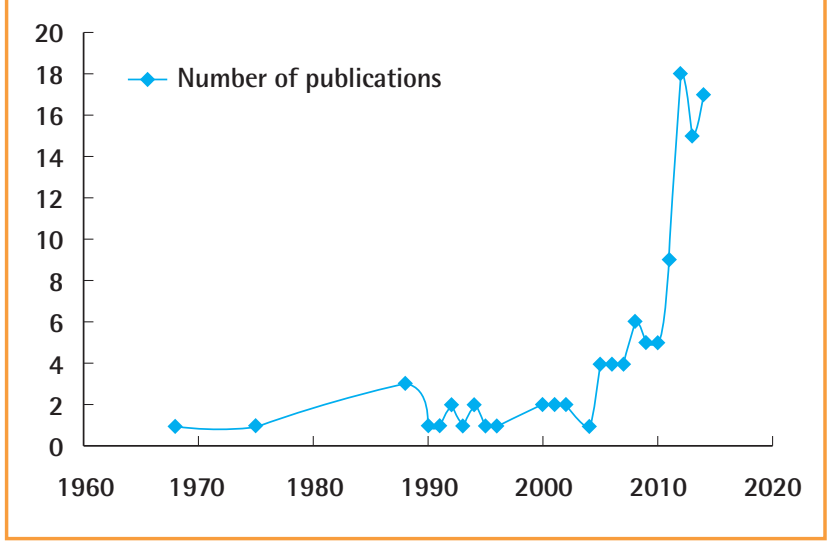

\section{Characteristics of the grafts}

$57 \%$ of studies reported injecting the graft in a single bolus technique, whereas $43 \%$ reported grafting in a fan-like pattern, distributing the graft in multiple passes using a single entry point underneath the skin (Fig. 5A). An average of $0.86 \pm 0.60 \mathrm{~mL}$ of fat was grafted in the studies; however volume of the graft could range from 0.10 to $1.50 \mathrm{~mL}$ in a single procedure (Fig. 5B).

\section{Duration of study and volume quantification}

Study duration and time of follow-up of graft volume retention ranged from 1 to 52 weeks. Most studies had more than one time point of sacrifice. The most common end points were 4 weeks (15\%), 8 weeks (11\%) and 12 weeks (19\%). One third of the studies had sacrifice time between $8-12$ weeks. $11 \%$ of studies lasted less than 1 week (Fig. 6).

Quantification of fat graft was done by 3 main approaches: weight $(63 \%)$ was the most popular modality and measured through a variety of scales. Some groups opted to use a pycnometer, which utilizes changes in pressure, and volume in a gas chamber to assess the volume and density of an explanted fat graft. $25 \%$ of studies used histology to quantify fat retention through the use of stains such as H\&E, Oil Red or Immunohistochemistry. Quantification based on digital imaging modalities was used in a $12 \%$ of the studies, either by micro-computed tomographic (CT) scanning or MRI (Fig. 7). More than one quantification method was used in the majority of the studies.

\section{DISCUSSION}

Fat grafting is a common component in many plastic surgery procedures completed today. According to the ASPS annual report, 67,609 procedures were performed in 2014 using fat as a

\section{Fig. 3. Breakdown of species utilized as well as mouse strain}

(A) Breakdown of species utilized more frequently. (B) Strain of mice more commonly selected.

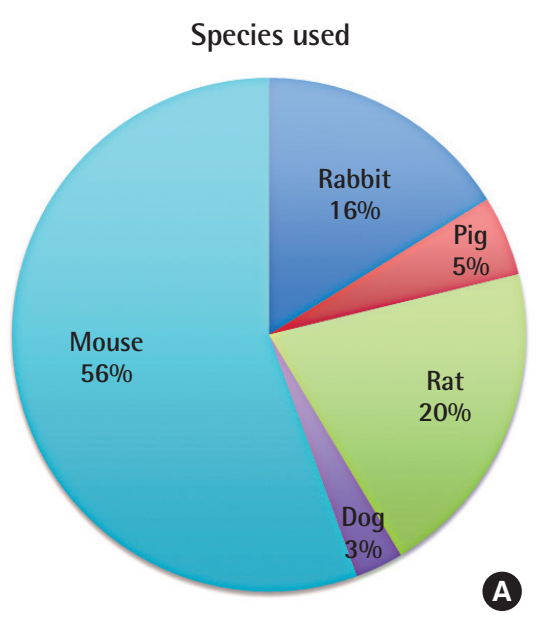




\section{Fig. 4. Grafts and recipient area in fat grafting studies}

(A) Type of graft more commonly used in studies: autologous (i.e., same mouse's groin to dorsum), human xenograft (i.e., human fat to mouse dorsum), other xenograft (i.e., rat to mouse), or allograft (i.e., mouse inguinal fat to another mouse's dorsum). (B) Recipient areas most frequently used. S0, subcutaneous.

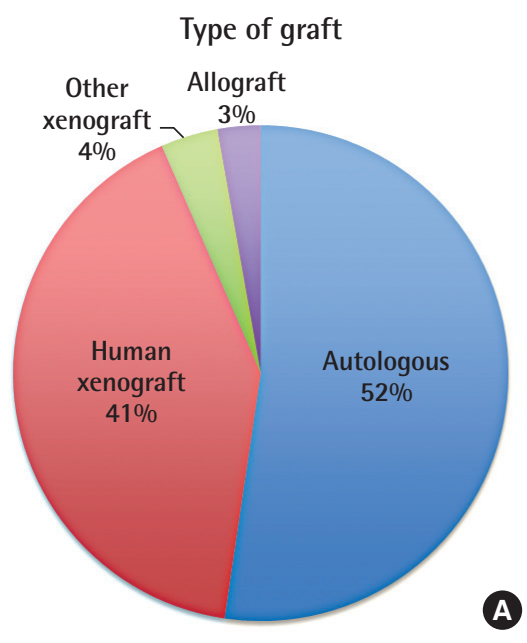

\section{Graft recipient area}

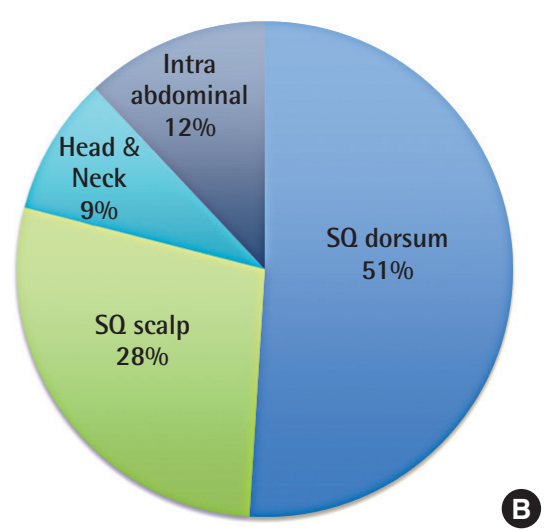

\section{Fig. 5. Technique for grafting and volume injected}

Technique used for grafting, as well as volume injected. SD, standard deviation.
Injection technique

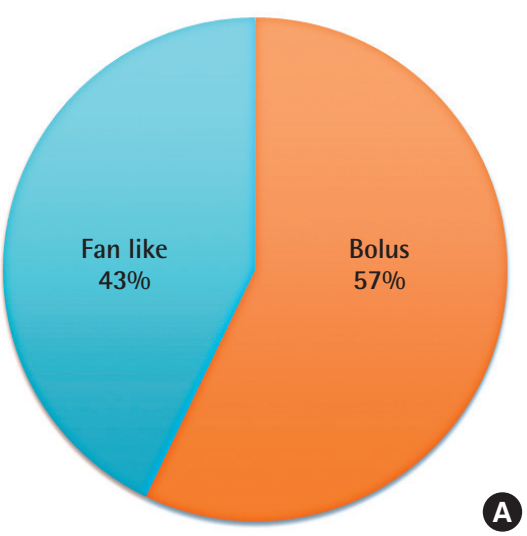

Grafted volume

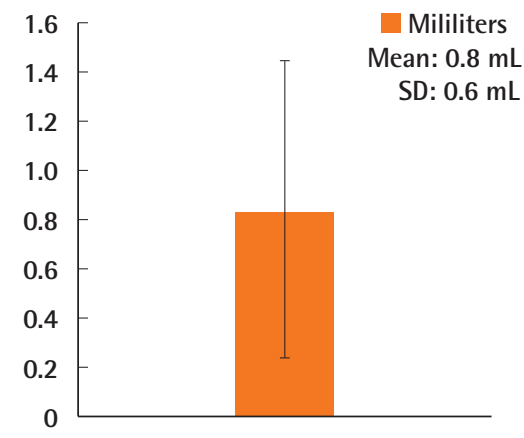

\section{Fig. 6. Duration of studies}

Overview of time-points used for sacrifice/volume quantification in studies.

Duration of studies in weeks

(Each study may have more than 1 time-point)

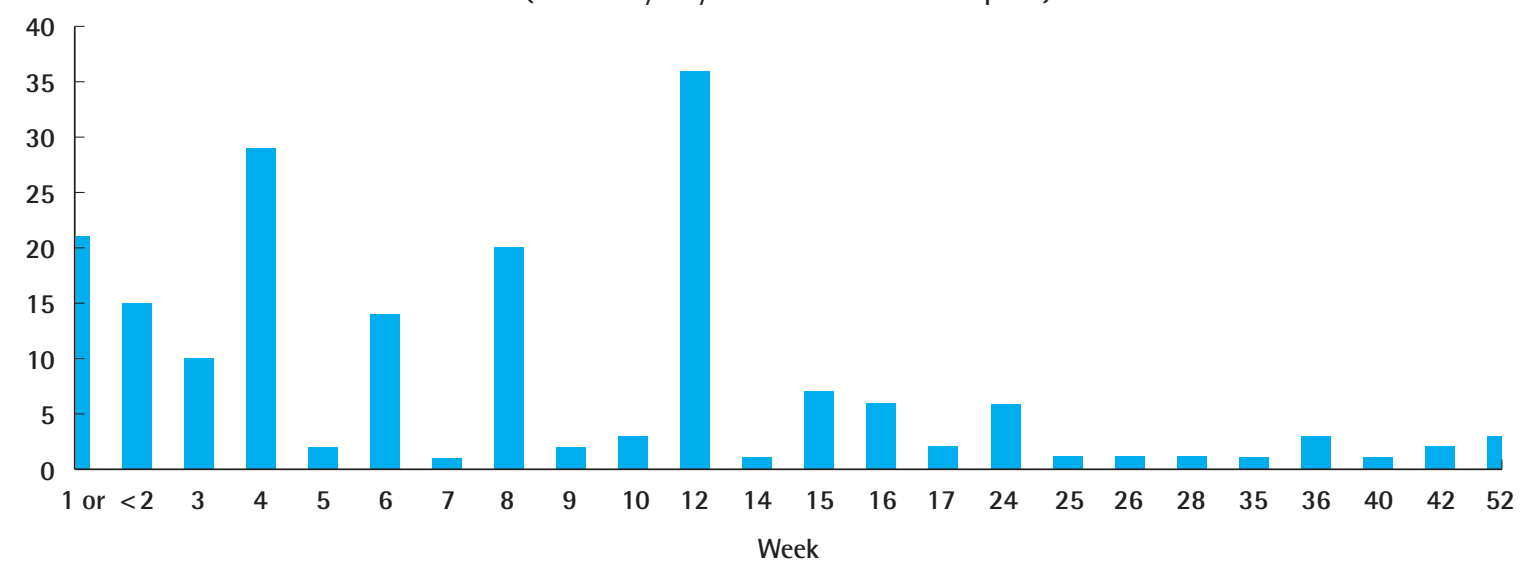




\section{Fig. 7. Graft volume quantification}

(A) Main methods for volume quantification used by the studies. (B) Staining techniques used in histologic sections by studies. $\mathrm{IHC}$, immunohistochemical.
Main method of graft quantification

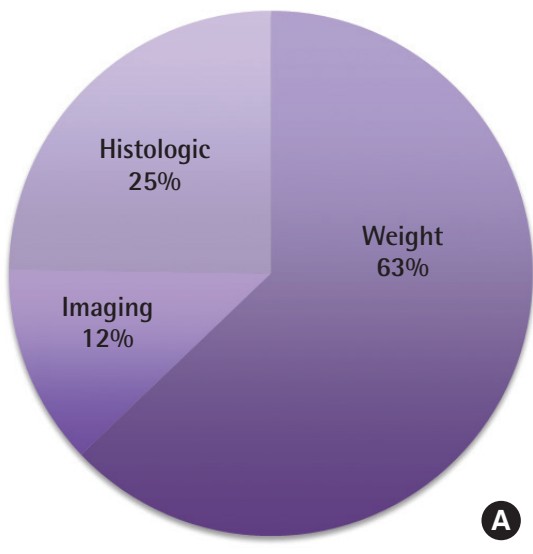

Quantification by histology

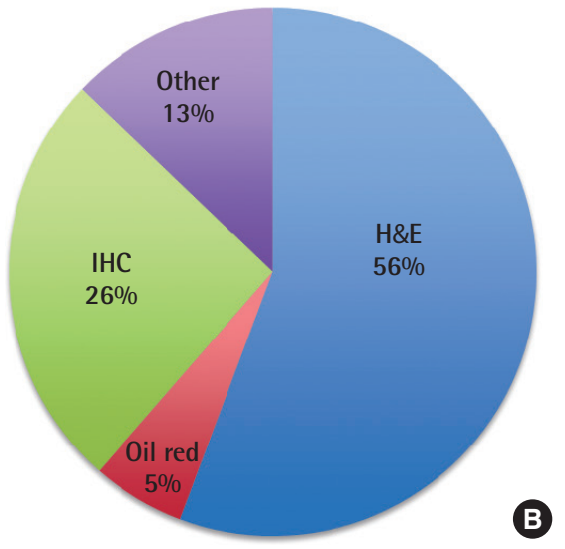

soft tissue filler [9]. The vast majority of plastic surgeons (85\%), utilize fat grafting, which justifies the $2 \%$ overall increase from 2013. In addition to cosmetic procedures, fat grafting is used in reconstructive procedures. Evidence supports an increasing prevalence of this technique across the spectrum of plastic surgery $[10]$.

When conducting translational studies, choosing the animal model is critical; It should match human characteristics as best as possible [11]. Mice are largely the most used model due to their extensive pedigree structure, large array of disease models, transgenic tools, knockout strains, and mouse-specific reagents [11]. Their skin shares some physiological similarities with humans, but also significant histological differences including the difference in thickness of epidermis and dermis, and the presence of the panniculus carnosus muscle between hypodermis and subcutaneous layer [12]. This is important as, in humans, superficial fat grafts are subdermal or subcutaneous. Due to the thin skin layers in mice, grafts are inevitably deposited in a deeper plane between the panniculus carnosus muscle and skeletal muscle. This same plane becomes a single unit and detaches from muscle once grafts are deposited, making it difficult for the graft to remain in the appropriate location and maintain shape.

Pig and human skin share the most histological similarities [13], having equally thick epidermis and dermis (human skin: $50-120 \mu \mathrm{m}$; pig skin: $30-140 \mu \mathrm{m})$. Pig's skin is strongly adhered to underlying internal structures and lacks the panniculus carnosus that rodents have. Additionally, the size and orientation of blood vessels, keratin proteins and a lipid film at the pigs skin's surface resemble human teguments.

However high costs and special care are involved in pig usage (\$275-\$1,321 each and \$11 per housing/day) making mouse (\$87-\$140 each and $\$ 0.70$ per housing/day) and rat (\$50-
$\$ 360$ each and $\$ 0.70$ per housing/day) models more popular. Therefore, should translational accuracy be jeopardized for economic reasons? As a counterpoint, pigs offer the advantage of a larger surface area, where multiple experiments can be carried in one single pig, and skin with human-like characteristics that could mimic the clinical setting better [14].

Studies are divided between using allografts, autografts or xenografts. Autografts avoid rejection problems while using inguinal fat, although some authors consider this tissue to be a mixture of brown-thermogenic fat and white-energy-storing adipose tissue $[15,16]$, which is known to have physiological differences compared to white subcutaneous fat [17]. On the other hand, xenografts utilize human tissue, but require a hindered immune system to avoid rejection, altering physiological variables that are not representative of a clinical setting. We found that few of the studies doing xenografts utilized non-immunocompromised animals, which was surprising for the authors as well, without giving a clear explanation to this phenomenon. It has been postulated that adipose tissue and adipose-derived stem cells have low immunogenicity. However this grants more research before doing xenografts in immunocompetent species. For now, finding a compromise between these approaches would be ideal. Perhaps an alternative could be doing autografts in a larger model where subcutaneous fat can be harvested by liposuction $[18,19]$.

Recipient site for fat grafts varies widely amongst research groups. Some groups prefer grafting below the scalp of mice, arguing that the lack of subcutaneous fat enables easier identification and cleaner resection at the time of explant $[20,21]$. On the other hand, proponents of grafting in the subcutaneous dorsum believe that it provides a more robust vasculature between 2 muscle layers (panniculus carnosus and the dorsal muscles) 


\section{Fig. 8. Histologic location of grafts}

Microscopic cross section of subcutaneous fat graft. Note the location under the dermis, between panniculus carnosus and the dorsal muscles. Stained with Perilipin-A IHC staining $(X 4)$ and counterstained with Hematoxylin. IHC, immunohistochemical.

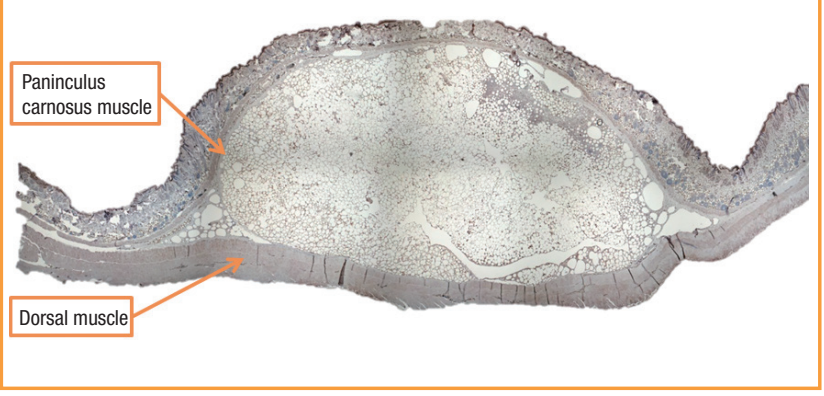

\section{Fig. 9. Splinting for graft protection}

A Rubber splint placed over the grafts for the first week post-injection to avoid migration and protect the graft from friction or selfinflicted damage.
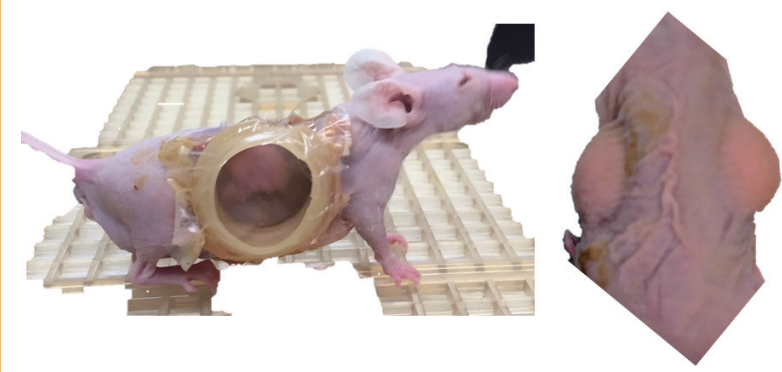

\section{Fig. 10. Quantification with micro-CT scanning}

Volume estimation using microCT scanning for small animals. Axial view and 3D reconstruction after contouring in computer software. CT, computed tomography.
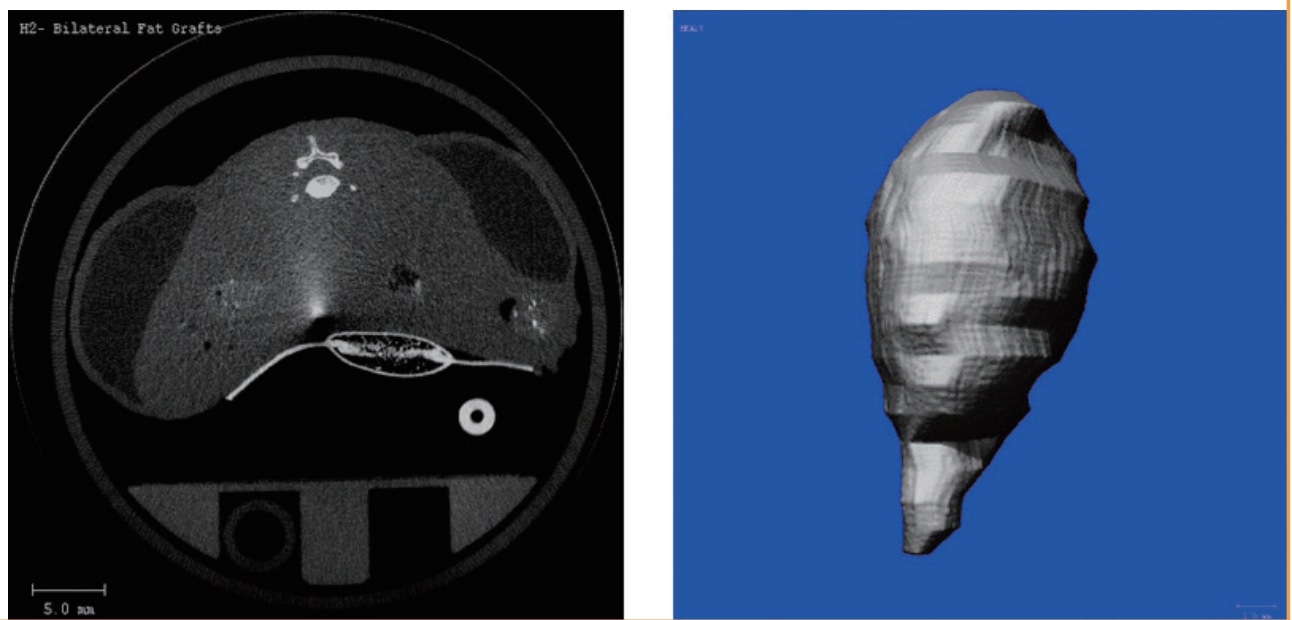

(Fig. 8) [22]. However, this may be more adventitious to fat graft survival when compared to the human situation. Graft survival requires a healthy perfusion of blood to ensure that vital oxygen diffusion occurs. The elasticity of skin and the resistance to expansion in response to an injection in a closed compartment results in an increase in interstitial pressure (i.e., breast, face), which effects fat survival [23]. However, small animals have loose skin, which alleviates the pressure under the graft. This allows for higher graft survival in mouse models. Therefore, in mice, more fat can be grafted in a bolus before reaching a critical pressure when compared to a similar amount in humans.

There is divided opinion between "bolus injection" and "fan like pattern". The latter might be more clinically translatable [22], however in our experience, the plane under the skin dissected with the injection cannula may act as a single plane (Fig. 8 ). This makes the fan pattern challenging. We believe that grafting in that way either makes the fat quantification difficult or the fat parcels coalesce under the plane where the graft is deposited (between panniculus carnosus and the dorsal muscles) [22], which defeats the purpose of grafting. Similarly, the graft is very mobile which is why, in our lab, we have opted for using external splints surrounding the grafts (Fig. 9). Regardless of the technique, we found that studies inject varying levels of fat volume (0.2-1.4 $\mathrm{mL}$ per graft).

The ideal time to follow-up grafts before sacrifice also remains undetermined. We found studies can range from 1-52 weeks, although $1 / 3$ of the articles believe the best window for sacrifice occurs between 8-12 weeks after the initial injection, once remodeling of the graft has occurred. This lack of standardization makes it difficult to compare studies to each other. Moreover, the evidence and the trend in the findings suggests that final changes in volume seem to stabilize after 8 weeks $[20,24]$ for a significant time, making this time-point potentially the earliest most cost-effective approach. 


\section{Fig. 11. Adipose tissue staining}

Perilipin-A is a reliable way to stain live and metabolically active adipocytes using IHC on paraffin embedded sections (Perilipin- $A$, $\mathrm{ICH}$ staining, $\times 20)$. IHC, immunohistochemical.

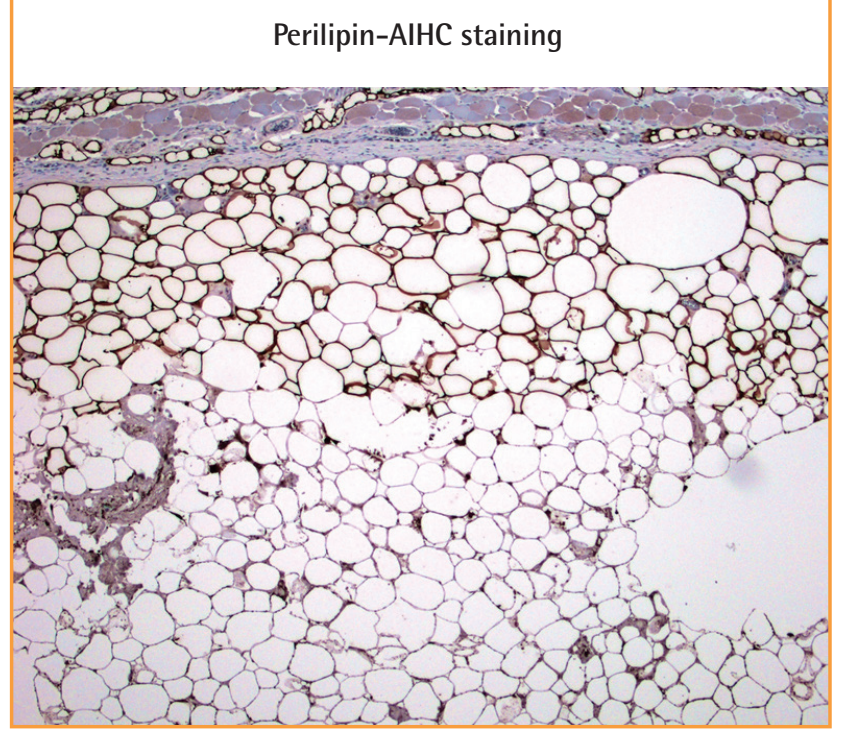

Different methods of quantification for final graft volume have been described. Weight estimation strategies are effective and have been used by the majority of the studies. Weight can be obtained by resecting the fat pad at the time of sacrifice [25], and then weighing it on an electronic scale [26]. Some groups will wash, blot or dry the fat after resection [26]. Others use fluid (saline/water) displacement to measure the volume of the fat pad. Some groups have opted for using a sophisticated Pycnometer [27] which measures volume and density of the fat pad inside a gas chamber. This device can cost around $\$ 11,462$ according to a distribution source. All these measuring techniques carry the risk of allowing for operator-dependent bias, as the weights are small and the scales are extremely sensitive. Small actions such as under-excision or over-excision of surrounding non-adipose tissue could severely skew the results. Additionally, they usually require the mouse to be sacrificed during the procedure.

Other imaging techniques such as micro-CT scanning can be a reliable way to obtain a 3D visualization of the graft and accurate volume estimation without sacrificing the animal (Fig. 10) $[20,24]$. Not all facilities have access to an in-vivo CT scanning machine for small species and costs can be expensive (at our institution the fee per scan is $\$ 100$ ). If the machine is not available, purchasing a micro-CT scanning machine can cost more than $\$ 370,000$, plus maintenance fees and trained personnel. In our opinion, both techniques quantify only mass and are equally effective, although we favor CT scanning as we believe has less room for technical error. However cross sectional histology

\section{Fig. 12. Evidence-based fat grafting model}

Our mouse model based on our experience and the evidence collected in the creation of this study. CT, computed tomographic; IHC, immunohistochemical. Mouse strain: 6-8 week old CD-1 Nude mice (Hairless, Athymic, mature T-
Cell deficient. Charles River Laboratories)

Fat specimen: Excised pannus from post-bariatric surgery patient. Pannus is located on table and Dry Liposuction from pannus is performed using a 3hole $2 \mathrm{~mm}$ Mentor cannula with multiple fan-like passes, using a $40 \mathrm{~mL}$ syringe with the plunger out at $10 \mathrm{~mL}$ mark.

Fat preparation: Fat is transferred to $10 \mathrm{~mL}$ syringes, centrifuged at $3,000 \mathrm{rpm}$ for 3 minutes. The upper oily layer is decanted, middle adipose part preserved and the bottom aqueous layer discarded. High-density fat is transferred (lower third of the adipose layer) using a 3-way stopcock and loaded into $1 \mathrm{~mL}$ syringes with luer lock.

Grafting: Using a $16 \mathrm{G}$ angio-catheter, a tunnel is created under the skin. Once the sharp part of the catheter penetrates the skin, the sharp portion is retracted and the sheath is advanced towards the desired area by blunt dissection. This will create a tunnel and open the plane between Panniculus Carnosus and skeletal muscle (Fig. 8).

Then fat is deposited. In our model we do bilateral grafts in order to keep opposite side as control. Right after grafting a donut-shaped rubber splint is placed on top and around the graft and held in position with Tegaderm (Fig. 9). Splints are kept in place for the first week post grafting in order to prevent migration, shear stress or self-inflicted damage to the graft once the revascularization phase has started.

Quantification: Micro CT scanning at baseline (Post op day 3) and 8 weeks post grafting followed by $3 \mathrm{D}$ reconstruction and volume quantification (Scanco Medical) (Fig. 10). Then grafts are explanted, formalin-fixed, paraffin-embedded and later stained for Perilipin-A by IHC and Hematoxylin and Eosin for quantification (Fig. 11).

becomes indispensable to define what the tissue is and assess the presence of cysts, calcifications and graft viability, as weight or 3D image do not distinguish what is inside of the graft. Therefore we found that almost every study used histological analysis in addition to their volume or mass quantification. Some groups have opted for Hematoxylin and Eosin or Oil Red staining. Others have preferred immunohistochemical (IHC) Perilipin-A staining [28], which is a surface marker that is present in live metabolically active adipocytes (Fig. 11). In our opinion, Perilipin-A, is more technically challenging, but is more specific in detecting live adipocytes, in comparison to $\mathrm{H} \& \mathrm{E}$ or oil red, which stains all structures equally, regardless of their metabolic status.

Our mouse model (Fig. 12).

\section{CONCLUSIONS}

In conclusion, studies involving fat grafting research in animal models are heterogeneous. At this point in time, performing xenografts from human fat on immunodeficient mice is the preferred animal model to assess volume retention of fat grafts. Almost all of the design parameters need to be standardized in or- 
der to achieve a systematic method and make results comparable. To our knowledge, this is the first study that provides an overview of this topic. Moreover, at least theoretically, scaling up to larger animals should be the goal of future research in fat grafting in order to have better translatable results for human studies. We hope our work can help promoting dialogue among the adipocyte research community to establish agreed upon parameters for research.

\section{REFERENCES}

1. Mazzola RF, Mazzola IC. History of fat grafting: from ram fat to stem cells. Clin Plast Surg 2015;42:147-53.

2. Zuk PA, Zhu M, Mizuno H, et al. Multilineage cells from human adipose tissue: implications for cell-based therapies. Tissue Eng 2001; 7:211-28.

3. Piccolo NS, Piccolo MS, Piccolo MT. Fat grafting for treatment of burns, burn scars, and other difficult wounds. Clin Plast Surg 2015;42:263-83.

4. Petit JY, Maisonneuve P, Rotmensz N, et al. Safety of Lipofilling in Patients with Breast Cancer. Clin Plast Surg 2015; 42: 339-44.

5. Kronowitz SJ, Mandujano CC, Liu J, et al. Lipofilling of the breast does not increase the risk of recurrence of breast cancer: a matched controlled study. Plast Reconstr Surg 2016; 137:385-93.

6. Lin JY, Wang C, Pu LL. Can we standardize the techniques for fat grafting? Clin Plast Surg 2015;42:199-208.

7. Longaker MT, Aston SJ, Baker DC, et al. Fat transfer in 2014: what we do not know. Plast Reconstr Surg 2014;133:1305-7.

8. Pu LL, Yoshimura K, Coleman SR. Future perspectives of fat grafting. Clin Plast Surg 2015;42:389-94.

9. American Society of Plastic Surgeons. 2014 National Clearinghouse of Plastic Surgery Procedural Statistics [Internet]. Arlington Heights, IL: American Society of Plastic Surgeons; 2014 [cited 2017 Jun 5]. Available from: https:// www.plasticsurgery.org/news/plastic-surgery-statistics.

10. Kling RE, Mehrara BJ, Pusic AL, et al. Trends in autologous fat grafting to the breast: a national survey of the american society of plastic surgeons. Plast Reconstr Surg 2013;132:3546.

11. Grisolia S. Letter: Hypoxia, saffron, and cardiovascular disease. Lancet 1974;2:41-2.

12. Benavides F, Oberyszyn TM, VanBuskirk AM, et al. The hairless mouse in skin research. J Dermatol Sci 2009;53:10-8.

13. Sullivan TP, Eaglstein WH, Davis SC, et al. The pig as a model for human wound healing. Wound Repair Regen 2001; 9:66-76.
14. Jemec B. Pre-operative traumatic congestion and composite skin-fat grafting. An experimental study. Scand J Plast Reconstr Surg 1968;2:91-6.

15. Contreras GA, Lee YH, Mottillo EP, et al. Inducible brown adipocytes in subcutaneous inguinal white fat: the role of continuous sympathetic stimulation. Am J Physiol Endocrinol Metab 2014;307:E793-9.

16. Rosenwald M, Perdikari A, Rulicke T, et al. Bi-directional interconversion of brite and white adipocytes. Nat Cell Biol 2013;15:659-67.

17. Tervala TV, Gronroos TJ, Hartiala P, et al. Analysis of fat graft metabolic adaptation and vascularization using positron emission tomography-computed tomographic imaging. Plast Reconstr Surg 2014;133:291-9.

18. Dillerud E, Heden P. Circulation of blood and viability after blunt suction lipectomy in pig buttock flaps. Scand J Plast Reconstr Surg Hand Surg 1993;27:9-14.

19. Lequeux C, Oni G, Wong C, et al. Subcutaneous fat tissue engineering using autologous adipose-derived stem cells seeded onto a collagen scaffold. Plast Reconstr Surg 2012; 130:1208-17.

20. Chung CR, Tsuji K, Nifuji A, et al. Micro-CT evaluation of tooth, calvaria and mechanical stress-induced tooth movement in adult Runx2/Cbfa1 heterozygous knock-out mice.J Med Dent Sci 2004;51:105-13.

21. Ullmann Y, Shoshani O, Fodor A, et al. Searching for the favorable donor site for fat injection: in vivo study using the nude mice model. Dermatol Surg 2005;31:1304-7.

22. Thanik VD, Chang CC, Lerman OZ, et al. A murine model for studying diffusely injected human fat. Plast Reconstr Surg 2009; 124:74-81.

23. Khouri RKJr, Khouri RE, Lujan-Hernandez JR, et al. Diffusion and perfusion: the keys to fat grafting. Plast Reconstr Surg Glob Open 2014;2:e220.

24. Atashroo DA, Paik KJ, Chung MT, et al. Assessment of viability of human fat injection into nude mice with microcomputed tomography.J Vis Exp 2015:e52217.

25. Kononas TC, Bucky LP, Hurley C, et al. The fate of suctioned and surgically removed fat after reimplantation for soft-tissue augmentation: a volumetric and histologic study in the rabbit. Plast Reconstr Surg 1993;91:763-8.

26. Por YC, Yeow VK, Louri N, et al. Platelet-rich plasma has no effect on increasing free fat graft survival in the nude mouse. J Plast Reconstr Aesthet Surg 2009;62:1030-4.

27. Fisher C, Grahovac TL, Schafer ME, et al. Comparison of harvest and processing techniques for fat grafting and adipose stem cell isolation. Plast Reconstr Surg 2013;132:35161. 
28. Eto H, Kato H, Suga H, et al. The fate of adipocytes after nonvascularized fat grafting: evidence of early death and re- placement of adipocytes. Plast Reconstr Surg 2012;129: 1081-92. 